\title{
Correlation of CD146 expression and clinicopathological characteristics in esophageal squamous cell carcinoma
}

\author{
YAN LI ${ }^{1,2}$, JIN-MING YU ${ }^{1}$, XUE-MEI ZHAN ${ }^{3}$, LI-LI LIU ${ }^{3}$, NING JIN $^{3}$ and YAN-XIA ZHANG ${ }^{2}$ \\ ${ }^{1}$ Department of Radiation Oncology, Shandong Cancer Hospital, Shandong University, Jinan, Shandong 250117; \\ ${ }^{2}$ Department of Radiation Oncology and ${ }^{3}$ Department of Pathology, Linyi People's Hospital, \\ Linyi, Shandong 276000, P.R. China
}

Received August 27, 2013; Accepted March 24, 2014

DOI: 110.3892/ol.2014.2227

\begin{abstract}
CD146, a cell adhesion molecule, is found in normal and tumor tissues. The level of its expression has been found to directly correlate with tumor progression and metastatic potential. The objective of this study was to investigate the expression of CD146 in esophageal squamous cell carcinoma (ESCC) and its correlation with clinicopathological parameters. Tumor specimens were collected from 63 patients with ESCC who underwent complete resection. We analyzed the CD146 expression levels in ESCC by immunohistochemistry. The expression of CD146 was detected and it was observed to correlate with clinicopathological parameters. Sixty-three cases of normal squamous mucosa were included for comparison. CD146 expression was identified in 46.0\% (29/63) of the ESCC samples, and no positive (weak to moderate or moderate to strong) expression was found in the normal squamous epithelium samples $\left(\chi^{2}=27.248 ; \mathrm{P}<0.0001\right)$. CD146 expression was associated with lymph node metastasis $\left(\chi^{2}=5.117 ; \mathrm{P}=0.024\right)$ and advanced clinical stage $\left(\chi^{2}=4.661 ; \mathrm{P}=0.031\right)$. CD146 expression was one of the significant predictors of survival (hazard ratio, 2.838; 95\% confidence interval 1.102-7.305). The overexpression of the CD146 gene was one of the important phenotypes and characteristics in ESCC carcinomatous change. We found that CD146 expression was associated with lymph node metastasis and advanced clinical stage, and was an indicator of poor prognosis in ESCC patients. CD146 may prove to be an important tumor marker for the individualized treatment for ESCC.
\end{abstract}

\section{Introduction}

Esophageal carcinoma is one of the most fatal types of cancer with highly aggressive potency. Due to its poor

Correspondence to: Professor Jin-ming Yu, Department of Radiation Oncology, Shandong Cancer Hospital, 440 Jiyan Road, Jinan, Shandong 250117, P.R. China

E-mail: yujinmingsd@yahoo.com.cn

Key words: CD146, esophageal squamous cell cancer, clinicopathological characteristics, immunohistochemistry prognosis and high incidence rate, which exceeds 100 cases per 100,000 individuals per year in China (1). it is important to investigate the initiation and progression of esophageal squamous cell carcinoma (ESCC) and to study the associated prognostic factors. Numerous genes and proteins with abnormal expression and function have been investigated in the pathogenesis of ESCC, such as epidermal growth factor receptor, survivin and cyclooxygenase-2 (2-4). However, the molecular mechanisms involved in the pathogenesis of ESCC are not fully understood. CD146, a member of the immunoglobulin gene superfamily, was first identified as a cell adhesion molecule and a marker of melanoma progression and metastasis (5). It has been demonstrated that CD146 is expressed on vascular endothelium, smooth muscle and other cells in normal tissue, and mediates cation-independent adhesion through interactions with an unidentified ligand on the surface of various cells (6).

Overexpression of CD146 has been identified in a number of types of cancer, including melanoma, prostate cancer, epithelial ovarian cancer and breast cancer (7-10). Its expression levels have been found to correlate with tumor progression and metastatic potential, thus establishing CD146 as an important candidate molecule involved in tumor growth and metastasis. However, at present, studies that report CD146 expression in ESCC patients are rare. Therefore, we evaluated the expression of CD146 in ESCC and its association with clinicopathological parameters, such as clinical stage of the disease, in the present study.

\section{Materials and methods}

Patients and specimens. The expression of CD146 in 63 surgically resected ESCC specimens and in 63 normal esophageal mucosa samples obtained from ESCC patients was analyzed by immunohistochemistry. In total, 63 patients with ESCC underwent surgery at Linyi People's Hospital (Linyi, China), from August 2010 to February 2012. All patients underwent total esophagectomy and radical lymph node dissection. Normal esophageal mucosal samples were taken from a region $>5 \mathrm{~cm}$ distant from the cancer, as non-tumor control samples. There were 63 cases in the control group. Histopathological specimens were fixed in $10 \%$ buffered formalin, processed routinely and embedded in paraffin. All specimens were obtained from 
Table I. Correlation between CD146 expression and the clinicopathoclinical parameters in the ESCC group.

\begin{tabular}{|c|c|c|c|c|}
\hline Parameters & $\mathrm{CD} 146^{+}$ & & CD146- & P-value \\
\hline \multicolumn{5}{|l|}{ Age, years } \\
\hline Range & & $41-77$ & & \\
\hline Mean & & 62.4 & & \\
\hline \multicolumn{5}{|l|}{ Gender, $\mathrm{n}$} \\
\hline Male & & 60 & & \\
\hline Female & & 3 & & \\
\hline Tumor size, $\mathrm{n}$ & & & & 0.309 \\
\hline$\leq 3 \mathrm{~cm}$ & 5 & & 10 & \\
\hline $3.1-6 \mathrm{~cm}$ & 17 & & 20 & \\
\hline$>6 \mathrm{~cm}$ & 7 & & 4 & \\
\hline Invasion depth ${ }^{\mathrm{a}}, \mathrm{n}$ & & & & 0.327 \\
\hline $\mathrm{T} 2$ & 4 & & 8 & \\
\hline $\mathrm{T} 3$ & 25 & & 26 & \\
\hline Differentiation status $^{\mathrm{b}}, \mathrm{n}$ & & & & 0.372 \\
\hline Low & 5 & & 4 & \\
\hline Moderate & 19 & & 19 & \\
\hline High & 5 & & 11 & \\
\hline Lymph node metastasis, $\mathrm{n}$ & & & & 0.024 \\
\hline No & 8 & & 19 & \\
\hline Yes & 21 & & 15 & \\
\hline Clinical stage $^{c}, n$ & & & & 0.031 \\
\hline II & 10 & & 21 & \\
\hline III & 19 & & 13 & \\
\hline
\end{tabular}

${ }^{a}$ According to the Internation Union against Cancer 2002 (12); baccording to World Health organization criteria 2000 (11); and ${ }^{\text {caccording to }}$ the tumor-node-metastasis classification system of the International Union against Cancer (12). ESCC, esophageal squamous cell carcinoma.

patients who had not received chemotherapy and radiotherapy prior to surgery. Following hematoxylin and eosin staining, all sections were reviewed and reexamined. The grade of tumor differentiation was determined according to the classification of the World Health Organization 2011 (11), and the clinical stage was according to the tumor-node-metastasis classification system of the International Union Against Cancer 2002 (12). The invasion depth was determined according to the criteria of the International Union Against Cancer 2002 (12). The study was approved by the ethics committee of Shandong Cancer Hospital of Shandong University (Shandong, China). Patients provided written informed consent.

Immunohistochemical staining. The specimens of ESCC and non-cancerous esophageal mucosa were cut into 4- to $5-\mu \mathrm{m}$-thick sections and mounted onto slides, deparaffinized with xylene, and rehydrated with graded concentrations of ethanol. Endogenous peroxidase activity was blocked with $3 \%$ hydrogen peroxide $\left(\mathrm{H}_{2} \mathrm{O}_{2}\right)$ in deionized water for $10 \mathrm{~min}$. The slides were then washed three times with phosphate-buffered saline (PBS, $\mathrm{pH}$ 7.2-7.4) buffer for $2 \mathrm{~min}$. An antigen retrieval technique was used before application of the primary antibody $(10 \mathrm{mmol} / 1$ sodium citrate solution, $\mathrm{pH} 6.0$ in a pressure cooker for 2-2.5 min). After three washes with PBS, an aliquot of $50 \mu \mathrm{l}$ of primary antibody (rabbit anti-human CD146 monoclonal antibody; ncl-cd146; Leica Biosystems, Newcastle Upon Tyne, United Kingdom) was applied to each section and incubated at $37^{\circ} \mathrm{C}$ for $60 \mathrm{~min}$. This was followed by washing three times with PBS, and the antibodies were detected using the secondary antibody detection kit Polink-1 PV-6000 (Zhongshan Goldenbridge Biotechnology Co., Ltd., Beijing, China). Sections were stained with 3,3'-diaminobenzidine (DAB) followed by distilled water. The sections were lightly counterstained in Haris hematoxylin solution (Zhongshan Goldenbridge Biotechnology Co., Ltd.) for microscopic examination (BX53, Olympus Corporation, Tokyo, Japan). The section were dehydration in an alcohol gradient, cleared with xylene and mounted using neutral gum.

Simultaneously, each section was incubated with PBS instead of the primary antibody as an internal negative control. The immunostained specimens were analyzed by two independent pathologists who were blinded to the patients' clinicopathological characteristics. Cytoplasm and membrane staining (brown reaction product) was regarded as a positive staining result for CD146. Five fields in each cancer and non-cancer section were evaluated at high power $(\mathrm{x} 400)$ to determine the proportion of immunostained tumor cells and the staining intensity of the cytoplasm and membrane in the entire sections. The staining 


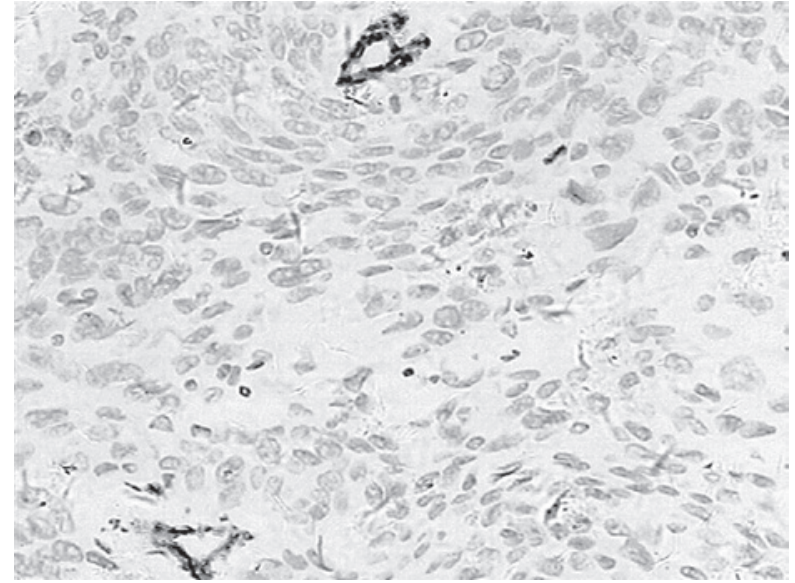

Figure 1. Representative image of negative immunohistochemical staining for CD146 (score of 1) in an esophageal squamous cell carcinoma specimen (magnification, $\mathrm{x} 200$ ).

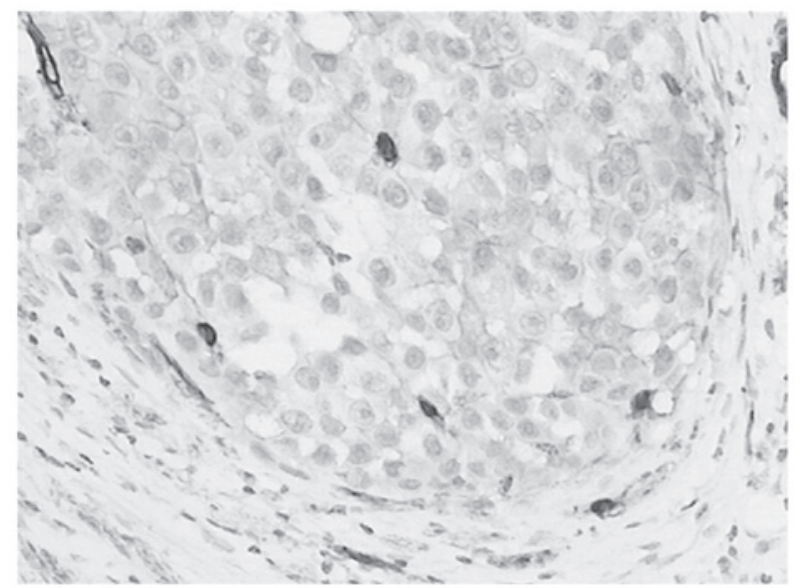

Figure 2. Representative image of weak immunohistochemical staining for CD146 (score of 1) in an esophageal squamous cell carcinoma specimen (magnification, $\mathrm{x} 200$ ).

strength was graded from 1 to $3: 1$, no positive staining or a weak staining (Figs. 1 and 2); 2, weak to moderate staining (Fig 3); 3, moderate to strong staining (Fig. 4). The case with positive cells $\geq 25 \%$ and/or scores $\geq 2$ was considered to be positive (13). At least five fields were observed, the average score in each tumor and non-tumor sections served as the result. All sections were scored twice to confirm the reproducibility of the results, and the highest score from the two observers was reported.

Follow-up. The patients were followed up every 3 months for the first year and then every 6 months for the next 2 years. The total follow-up period was defined as the time from diagnosis to the date of death or the last follow-up appointment if patients remained alive. All 63 patients were included in survival data analysis. The last follow-up appointment was carried out in May 2013, with a mean observation period of 17.6 months (range, 3-35 months).

Statistical analysis. All calculations were performed using SPSS software, version 16.0 (SPSS, Inc., Chicago, IL, USA). The associations between CD146 expression and clinicopathological

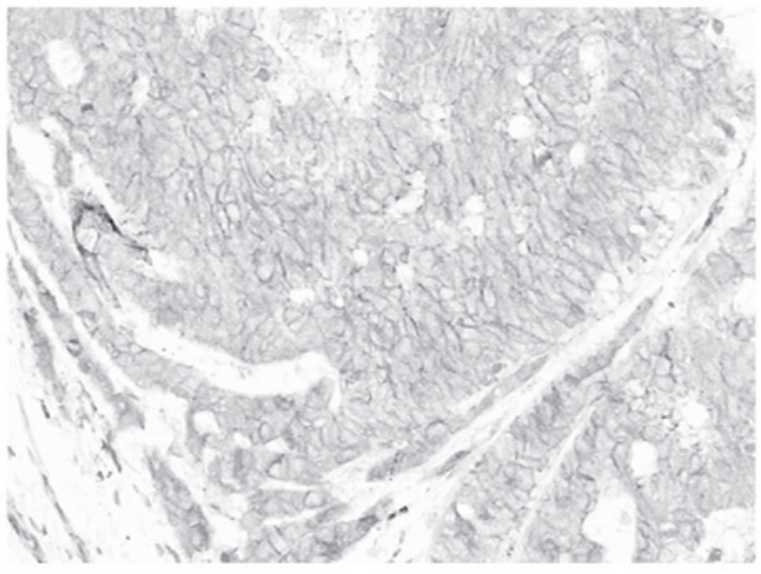

Figure 3. Representative image of weak to moderate immunohistochemical staining for CD146 (score of 2) in an esophageal squamous cell carcinoma specimen (magnification, $\mathrm{x} 200$ ).

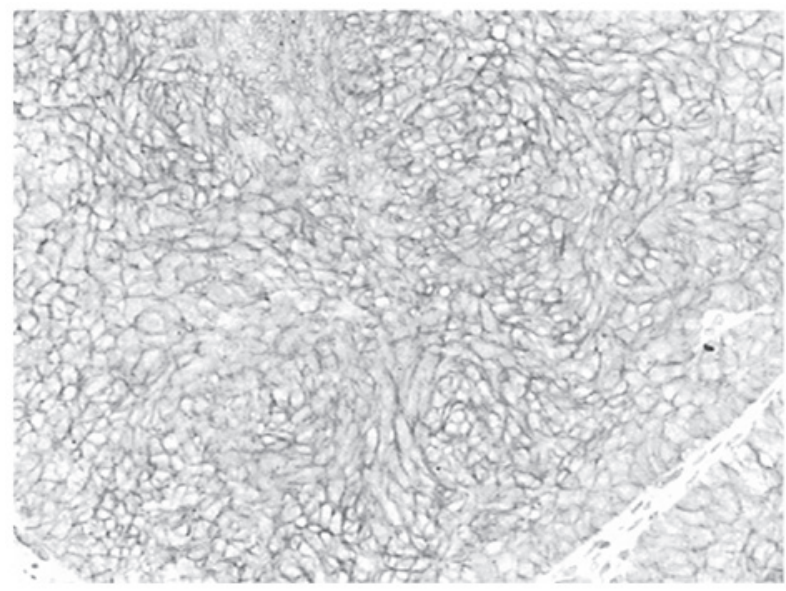

Figure 4. Representative image of moderate to strong immunohistochemical staining for CD146 (score of 3) in an esophageal squamous cell carcinoma specimen (magnification, $\mathrm{x} 200$ ).

variables were assessed using the $\chi^{2}$ and Fisher's exact tests. The association between CD146 expression and survival time was assessed using the Cox regression model. $\mathrm{P}<0.05$ was considered to indicate a statistically significant difference.

\section{Results}

Immunohistochemistry. Immunohistochemistry revealed that CD146-positive staining was localized in the membrane and cytoplasm of tumor cells in the tumor tissues. CD146 expression was identified in $46.0 \%(29 / 63)$ of the ESCC samples, and no positive (weak to moderate or moderate to strong)expression was found in the 63 normal squamous epithelium samples (Fig. 5) $\left(\chi^{2}=27.248 \mathrm{P}<0.0001\right)$. The negative control group was underwent the same steps as previously described, with the exception that the CD146 antibody was replaced with PBS (Fig. 6).

Associations between CD146 expression and clinicopathological variables. The associations between CD146 expression and clinicopathological variables were investigated. Positive expression of CD146 was found in $46.0 \%$ (29/63) of the ESCC 


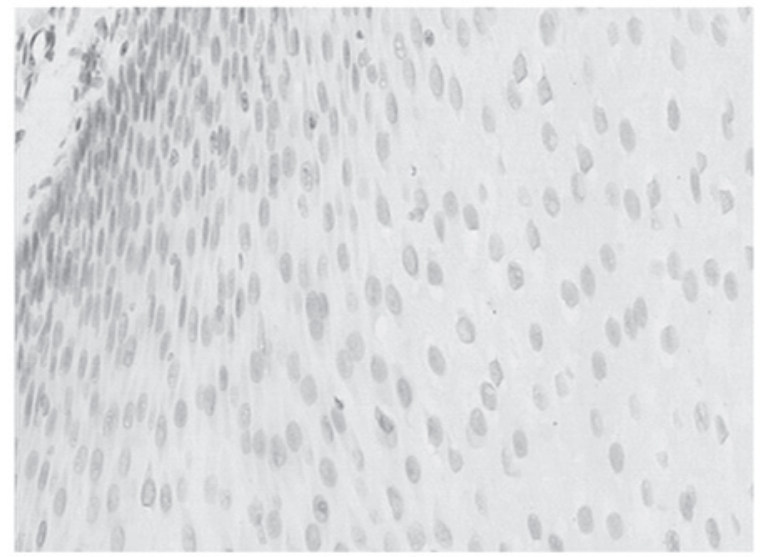

Figure 5. Representative image of negative immunohistochemical staining for CD146 (score of 1) in a normal esophageal mucosa sample (magnification, x200).

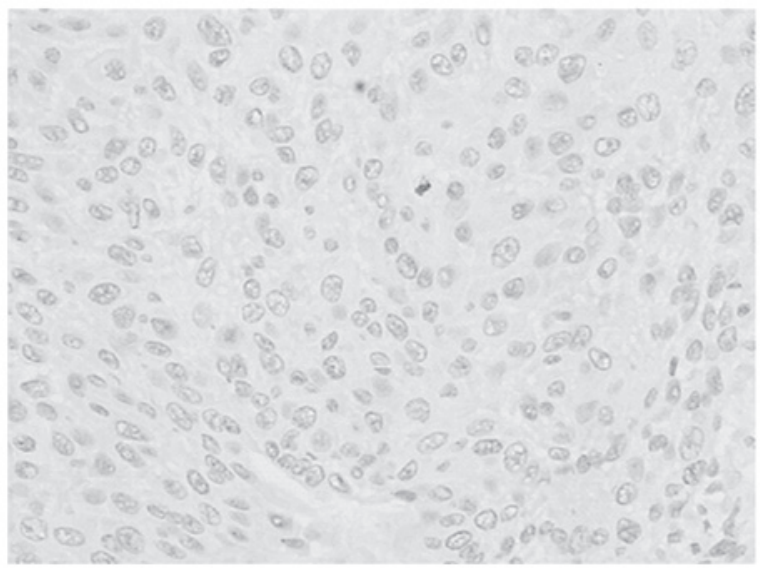

Figure 6. Representative image of phosphate-buffered saline instead of the primary antibody being used as an internal negative control in an esophageal squamous cell carcinoma specimen (magnification, x200).

samples, which was significantly higher than that in the normal esophageal epithelium samples which demonstrated no immunostaining $\left(\chi^{2}=27.248 ; \mathrm{P}<0.0001\right)$. CD146 expression was associated with lymph node metastasis $\left(\chi^{2}=5.117\right.$; $\mathrm{P}=0.024)$ and advanced clinical stage $\left(\chi^{2}=4.661 ; \mathrm{P}=0.031\right)$. No correlation was found with tumor size $\left(\chi^{2}=2.346 ; \mathrm{P}=0.309\right)$, invasion depth $\left(\chi^{2}=0.962 ; \mathrm{P}=0.327\right)$ or tumor differentiation status $\left(\chi^{2}=1.977 ; \mathrm{P}=0.372\right)$ (Table I).

Association between CD146 expression and survival time. The mean survival time of patients with positive CD146 expression was 15 months while that of patients with negative CD146 expression was 25 months. In the multivariate analysis, the association between CD146 expression and survival time was statistically significant (HR, 2.838; 95\% CI: 1.102-7.305; P=0.031) (Fig. 7).

\section{Discussion}

CD146 is a cell-cell or cell-matrix adhesion molecule that was first described in melanoma (5). Previous studies have indicated that CD146 expression correlates with the malignant progression and metastatic potential of human melanoma

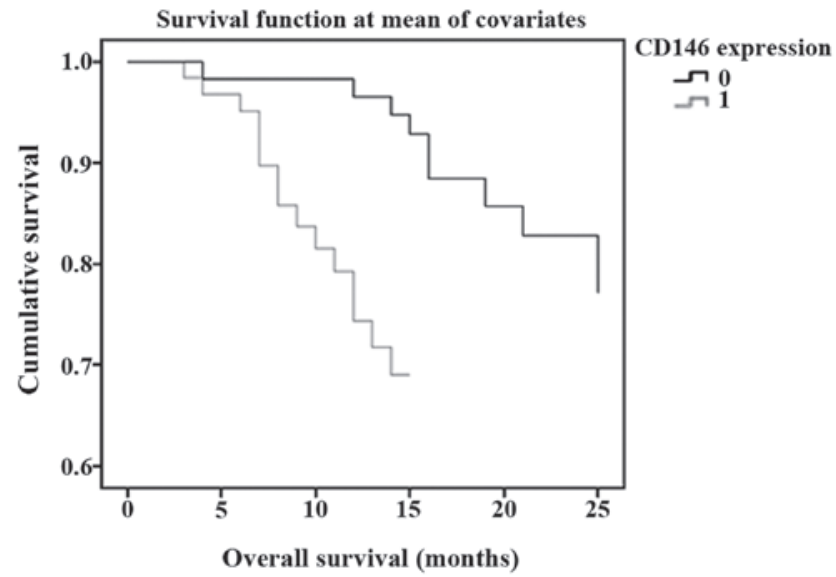

Figure 7. Correlation between CD146 expression and survival time in esophageal squamous cell carcinoma.

cells (14-18). Expression of CD146 has been observed in certain normal human tissues and numerous malignancies, such as non-small cell lung cancer, gallbladder adenocarcinoma and gastric cancer $(13,19,20)$. The current study demonstrated that CD146 expression was significantly higher in ESCC than in the normal esophageal mucosal tissue. Additionally, it was identified that CD146 expression was associated with lymph node metastasis $(\mathrm{P}=0.024)$ and advanced clinical stage $(\mathrm{P}=0.031)$ in ESCC. However, no correlations between $\mathrm{CD} 146$ expression and tumor size $(\mathrm{P}=0.309)$, invasion depth $(\mathrm{P}=0.327)$ and differentiation grading $(\mathrm{P}=0.372)$ were identified. The results shown in Table I demonstrate that five of the nine low differentiation patients, 19 of the 38 moderate differentiation patients and five of the 16 high differentiation patients were positive for immunohistochemical expression. In addition, the results showed that in tumors with low and intermediate differentiation, the expression of CD146 was higher than that in highly differentiated tumors (55.6, 50.0 and $31.3 \%$ respectively), although the differences were not statistically significant. Similarly, 4 of the $12 \mathrm{~T} 2$ patients and 25 of the $51 \mathrm{~T} 3$ patients were positive for immunohistochemical expression, while five of the 15 patients with tumor sizes of $\leq 3 \mathrm{~cm}$, 17 of the 37 patients with tumor sizes of 3.1-6 $\mathrm{cm}$ and 7 of the 11 patients with tumor sizes of $>6 \mathrm{~cm}$ were positive for immunohistochemical expression. CD146 expression in T3 tumors (49.0\%) was higher than that in T2 tumors (33.3\%), and the levels of expression increased with an increase in tumor size (33.3, 45.9, 63.6\%), although the results were not statistically significant. The results of the present study demonstrated that CD146 may have a role in malignant progression in esophageal squamous cell carcinoma and may be associated with a more aggressive tumor phenotype. Liu et al (20) reported that CD146 expression correlated positively with lymph node involvement in gastric cancer patients. The results of the present study are consistent with this finding in gastric cancer.

CD146 expression has been found to be correlated with aggressiveness and development of metastasis, and is a predictor of worse prognosis in certain cancer types (21). Advanced tumor stage is an important prognostic factor for solid tumors. CD146 is associated with an advanced tumor stage in melanoma, prostate cancer, ovarian cancer and 
triple-negative breast cancer $(7-9,22)$. In the present study, CD146 was demonstrated to be associated with an advanced tumor stage in ESCC. Metastasis occurs through a series of steps, including local invasion, intravasation, transport, extravasation and colonization (23). Epithelial to mesenchymal transition is a process in which the epithelial cells lose polarity and develop a mesenchymal phenotype and has been implicated in the initiation of metastasis (22). CD146 is a component of the inter-endothelial junction (24), and is now recognized as a marker of mesenchymal cells (25). CD146 may directly or indirectly contribute to tumor aggressiveness by promoting malignant cell motility (10). The presence of lymph node metastasis is an important factor in the clinical evaluation of esophageal cancer patients (26). Lymphangiogenesis is a significant step in the lymphatic metastasis of tumors. Neonatal lymph vessels finally cause metastasis to regional lymph nodes. A previous study has found that lymph vessel density has a close association with progression, metastasis and prognosis of malignant tumors (13). A study by Sundar and Ganesan indicated that tumor-induced lymphangiogenesis was a predictive indicator of metastasis to lymph nodes (27). Tumor-secreted cytokines, such as vascular endothelial growth factors (VEGF)-C and -D, bind to VEGF receptors on lymphatic endothelial cells and induce proliferation and growth of new lymphatic capillaries. This process is similar to the well known mechanism of angiogenesis; the proliferation of new blood vessel capillaries (28). Luo et al (14) reported that CD146 directly interacts with actin-linking ezrin-radixin-moesin (ERM) proteins and recruits ERM proteins to cell protrusions, promoting the formation and elongation of microvilli and leading to cytoskeleton remodeling and finally cell migration. However, the exact molecular mechanism whereby CD146 is involved in lymph node metastasis remains unknown. Further studies are required to investigate this issue.

It is well acknowledged that advanced stage and lymph node metastasis are important prognostic factors for ESCC. The current study demonstrated that CD146 expression was associated with advanced clinical stage and lymph node metastasis in ESCC patients, and was therefore an indicator of poor prognosis in these patients. Overexpression of the CD146 gene was one of the important phenotypes and characteristics in ESCC carcinomatous change. This study suggests an important role for CD146 in the development of ESCC. CD146 may present as a potential therapeutic target for the individualized treatment of ESCC.

\section{References}

1. Li JS, Ying JM, Wang XM, et al: Promoter methylation of tumor suppressor genes in esophageal squamous cell carcinoma. Chin J Cancer 32: 3-11, 2013.

2. Sarbia M, Ott N, Pühringer-Oppermann F and Brucher BL: The predictive value of molecular markers (p53, EGFR, ATM, CHK2) in multimodally treated squamous cell carcinoma of the oesophagus. Br J Cancer 97: 1404-1408, 2007.

3. Li C, Li Z, Zhu M, et al: Clinicopathological and prognostic significance of survivin over-expression in patients with esophageal squamous cell carcinoma: a meta-analysis. PLoS One 7: e44764, 2012.

4. Kuo KT, Chow KC, Wu YC, et al: Clinicopathologic significance of cyclooxygenase-2 overexpression in esophageal squamous cell carcinoma. Ann Thorac Surg 76: 909-914, 2003.

5. Lehmann JM, Riethmuller G and Johnson JP: MUC18, a marker of tumor progression in human melanoma, shows sequence similarity to the neural cell adhesion molecules of the immunoglobulin superfamily. Proc Natl Acad Sci USA 86: 9891-9895, 1989.
6. Ouhtit A, Gaur RL, Abd Elmageed ZY, et al: Towards understanding the mode of action of the multifaceted cell adhesion receptor CD146. Biochim Biophys Acta 1795: 130-136, 2009.

7. Melnikova VO, Balasubramanian K, Villares GJ, et al: Crosstalk between protease-activated receptor 1 and platelet-activating factor receptor regulates melanoma cell adhesion molecule (MCAM/MUC18) expression and melanoma metastasis.J Biol Chem 284: 28845-28855, 2009.

8. Wu GJ, Peng Q, Fu P, et al: Ectopical expression of human MUC18 increases metastasis of human prostate cancer cells. Gene 327: 201-213, 2004.

9. Aldovini D, Demichelis F, Doglioni C, et al: M- CAM expression as marker of poor prognosis in epithelial ovarian cancer. Int $\mathrm{J}$ Cancer 119: 1920-1926, 2006.

10. Zabouo G, Imbert AM, Jacquemier J, et al: CD146 expression is associated with a poor prognosis in human breast tumors and with enhanced motility in breast cancer cell lines. Breast Cancer Res 11: R1, 2009.

11. Gabbert HZ, Shimoda T, Hainaut P, et al: Tumours of the oesophagus. In: World Health Organization Classification of Tumours: Pathology and Genetics of Tumours of the Digestive System. Hamilton SR and Alaltonen LA (eds). IARC Press, Lyon, pp10-16, 2000.

12. TNM Classification of the Esophagus. International Union Against Cancer. 6th edition. J Willy \& Sons, New York, NY, pp2-25, 2002.

13. Wang W, Yang ZL, Liu JQ, Jiang S and Miao XY: Identification of CD146 expression, angiogenesis, and lymphangiogenesis as progression, metastasis, and poor prognosis related markers for gallbladder adenocarcinoma. Tumour Biol 33: 173-182, 2012.

14. Luo Y, Zheng C, Zhang J, et al: Recognition of CD146 as an ERM-binding protein offers novel mechanisms for melanoma cell migration. Oncogene 31: 306-321, 2012.

15. Luca M, Hunt B, Bucana CD, et al: Direct correlation between MUC18 expression and metastatic potential of human melanoma cells. Melanoma Res 3: 35-41, 1993.

16. Johnson JP: Cell adhesion molecules in the development and progression of malignant melanoma. 18: 345-357, 1999.

17. Xie S, Luca M, Huang S, et al: Expression of MCAM/MUC18 by human melanoma cells leads to increased tumor growth and metastasis. Cancer Res 57: 2295-2303, 1997.

18. Zabouo G, Imbert AM, Jacquemier J, et al: CD146 expression is associated with a poor prognosis in human breast tumors and with enhanced motility in breast cancer cell lines. Breast Cancer Res 11: R1, 2009.

19. Kristiansen G, Yu Y, Schluns K, et al: Expression of the cell adhesion molecule CD146/MCAM in non-small cell lung cancer. Anal Cell Pathol 25: 77-81, 2003.

20. Liu WF, Ji SR, Sun JJ, et al: CD146 Expression correlates with epithelial-mesenchymal transition markers and a poor prognosis in gastric cancer. Int J Mol Sci 13: 6399-6406, 2012.

21. Shih I-M, Nesbit M, Herlyn M and Kurman RJ: A new Mel-CAM (CD146)-specific monoclonal antibody, MN-4, on paraffin-embedded tissue. Mod Pathol 11: 1098-1106, 1998.

22. Zeng Q, Li W, Lu D, et al: CD146, an epithelial-mesenchymal transition inducer, is associated with triple-negative breast cancer Proc Natl Acad Sci USA 109: 1127-1132, 2012.

23. Tsai JH and Yang J: Epithelial-mesenchymal plasticity in carcinoma metastasis Genes Dev 27: 2192-2206, 2013.

24. Bardin N, Anfosso F, Masse JM, et al: Identification of CD146 as a component of the endothelial junction involved in the control of cell-cell cohesion. Blood 98: 3677-3684, 2001.

25. Delorme B, Ringe J, Gallay N, et al: Specific plasma membrane protein phenotype of culture-amplified and native human bone marrow mesenchymal stem cells. Blood 111: 2631-2635, 2008.

26. Tanaka T, Ishiguro H, Kuwabara Y, et al: Vascular endothelial growth factor C (VEGF-C) in esophageal cancer correlates with lymph node metastasis and poor patient prognosis. J Exp Clin Cancer Res 29: 83, 2010.

27. Sundar SS and Ganesan TS: Role of lymphangiogenesis in cancer. J Clin Oncol 25: 4298-4207, 2007.

28. Nathanson SD: Insights into the mechanisms of lymph node metastasis. Cancer 98: 413-423, 2003. 